\title{
Measuring preferential attachment for evolving networks
}

\author{
H. Jeong, Z. Néda* and A.L. Barabási \\ University of Notre Dame, Department of Physics, Notre Dame, IN 46616, USA
}

(Last revised May 29, 2018)

\begin{abstract}
A key ingredient of current models proposed to capture the topological evolution of complex networks is the hypothesis that highly connected nodes increase their connectivity faster than their less connected peers, a phenomenon called preferential attachment. Measurements on four networks, namely the science citation network, Internet, actor collaboration and science coauthorship network indicate that the rate at which nodes acquire links depends on the node's degree, offering direct quantitative support for the presence of preferential attachment. We find that for the first two systems the attachment rate depends linearly on the node degree, while for the latter two the dependence follows a sublinear power law.

PACS numbers:89.65.-s, 89.75.-k, 05.10.-a
\end{abstract}

Modeling the highly interconnected nature of various social, biological and communication systems as complex networks or graphs has attracted much attention in the last few years. 114]. As for a long time these networks were modeled as completely random 15, 16, the recent interest is motivated by the increasing evidence that real network display short length-scale clustering [1,2] and obey unexpected scaling laws [3, [1], interpreted as signatures of deviation from randomness. Current approaches, using the tools of statistical physics [6, 8, 9 ] search for universalities both in the topology of these webs and in the dynamics governing their evolution. These efforts resulted in a class of models that view networks as evolving dynamical systems, rather than static graphs. Most evolving network models [4,66,9] are based on two ingredients [4]: growth and preferential attachment. The growth hypothesis suggests that networks continuously expand through the addition of new nodes and links between the nodes, while the preferential attachment hypothesis states that the rate $\Pi(k)$ with which a node with $k$ links acquires new links is a monotonically increasing function of $k$. While most versions of such evolving network models assume that $\Pi(k)$ is linear in $k$ [4, 5, 9], recently several authors proposed that $\Pi(k)$ could follow a power-law [8,10]. Consequently, the time evolution of the degree $k_{i}$ of node $i$ can be obtained from the first order differential equation

$$
\frac{d k_{i}}{d t}=m \Pi\left(k_{i}\right)
$$

where $m$ is a constant and $\Pi(k)$ has the form

$$
\Pi\left(k_{i}\right)=\frac{k_{i}^{\alpha}}{\sum_{j} k_{j}^{\alpha}}=C(t) k_{i}^{\alpha},
$$

with $\alpha>0$ an unknown scaling exponent. For $\alpha=1$ these models reduce to the scale-free model [4], for which the degree distribution $P(k)$, giving the probability that a node has $k$ links, follows $P(k) \propto k^{-\gamma}$ with $\gamma=3$. As Krapvisky, Redner and Leyvraz have shown [8], for $\alpha<1$ the degree distribution follows a stretched exponential, while for $\alpha>1$ a gelation-like phenomenon is expected, where a single site links to nearly all other nodes. On the other hand the hypothesis (2) raises a series of fundamental questions, that are not yet supported directly by experimental data. First, is preferential attachment indeed present in real networks? I.e. does indeed $\Pi(k)$ depend on $k$, or it is $k$ independent, as assumed both by the Erdős-Rényi [15] or Watts-Strogatz [2] models? Second, if $\Pi(k)$ does indeed depend on $k$, what is its functional form? Is it linear, as assumed in [4], or follows a power-law as suggested in $[8,10$ ? Could $\Pi(k)$ follow some unknown and yet unexplored functional form?

Here we offer the first direct attempt to answer these questions in quantitative terms by proposing a numerical method that allows us to extract the functional form of $\Pi(k)$ directly from dynamical data on real evolving networks. Our measurements indicate that $\Pi(k)$ follows a power law for all investigated networks. For the Internet and the citation network we find $\alpha=1$, while for the science collaboration network and the Hollywood actor network the results indicate sublinear attachment, i.e. $\alpha<1$. These results offer the crucial missing link for modeling the dynamics of complex evolving networks.

Methods: To measure $\Pi(k)$ we use computerized data on the dynamics of large networks. Consider a network for which we know the order in which each node and link joins the system. According to (1) and (2) the function $\Pi(k)$ gives the rate at which an existing node with $k$ links acquires new links as the network grows. To measure $\Pi(k)$ we need to monitor to which old node new nodes link to, in function of the degree of the old node. However, there is an important problem with this simple approach: the normalization constant, $C(t)$, depends on the time at which a given node joins the system, creating unwanted biases in the measurements. To avoid such bias we study the attachment of new nodes within a relatively short time-frame. Consider all nodes existing in the system at time $T_{0}$, called " $T_{0}$ nodes". Next select a group of " $T_{1}$ nodes", added between $\left[T_{1}, T_{1}+\Delta T\right]$, where 
$\Delta T<<T_{1}$ and $T_{1}>T_{0}$. When a $T_{1}$ node joins the system we record the degree $k$ of the $T_{0}$ node to which the new node links to. The histogram providing the number of links acquired by the $T_{0}$ nodes with exactly $k$ degree, after normalization, gives the $\Pi\left(k, T_{0}, T_{1}\right)$ function. If the growing network develops a stationary state, $\Pi\left(k, T_{0}, T_{1}\right)$ should be independent of $T_{0}$ and $T_{1}$, and should depend on $k$ only, providing us the $\Pi(k)$ preferential attachment function. As we are forced to use relatively short $\Delta T$ intervals, even for large networks with hundreds of thousands of nodes $\Pi(k)$ has significant fluctuations, particularly for large $k$. To reduce the noise level, instead of $\Pi(k)$ we study the cumulative function:

$$
\kappa(k)=\int_{0}^{k} \Pi(k) d k .
$$

If $\Pi(k)$ follows (2), we expect that

$$
\kappa(k) \propto k^{\alpha+1} .
$$

Measurements: The method described above can be applied to systems for which the order in which the nodes are added to the network is known. In this respect we had access to four different computerized networks, whose main parameters are shown in Table 1.

(1) In the coauthorship networks of neuro-science (NS) the nodes are scientists, two nodes being linked if they coauthored a paper together [11]. The database considered by us contains paper titles and authors of all relevant journals in the field of NS published between 1991-98. Similar to other collaboration networks 12 the distribution $P(k)$ for NS follows a power-law. Papers published between 1991-9x are used to reveal the network topology up to the considered $199 \mathrm{x}$ year, so that papers published in year $199 \mathrm{x}+1$ are used to measure $\Pi(k)$.

(2) In the citation network the nodes are papers published in 1988 in Physical Review Letters, and links represent the citations these articles received. $T_{0}$ is chosen as the year 1989 .

(3) In the actor network nodes are actors which are linked if they played together in a movie. The network investigated by us contains all movies and actors from 1892 up to 1999 [2, 18]. We determined $\Pi(k)$ for actors that debuted between 1920 and 1940, i.e. $T_{0}=1940$. We followed the evolution of the new links yearly between 1942 and 1993.

(4) For the Internet data we investigated the nodes represent Autonomous Systems (AS) and links are direct connections between them [19]. The available data follows the network evolution from 1997 up to the present days. The function $\Pi(k)$ was determined for the nodes existing in year 2000 .

Results: The $\kappa(k)$ functions obtained for the discussed databases are shown in Figs. 1 and 2. If preferential attachment is absent, i.e. $\Pi(k)$ is independent of $k$, we expect $\kappa(k) \propto k$. In Figs. 1 and 2 we show as continuous line the linear fit. In each of the investigated examples the increase of $\kappa(k)$ is faster than linear, offering direct evidence that preferential attachment is present in each of the considered systems. Furthermore, we find that the curves follow a straight line on a log-log plot, indicating that with a good approximation the power law hypothesis (2) is valid. Note that apart from statistical fluctuations, the functional form of $\Pi(k)$ is independent of $T_{0}$, supporting the stationary nature of the attachment process. There is some degree of variation, however, when it comes to the value of the exponent $\alpha$.
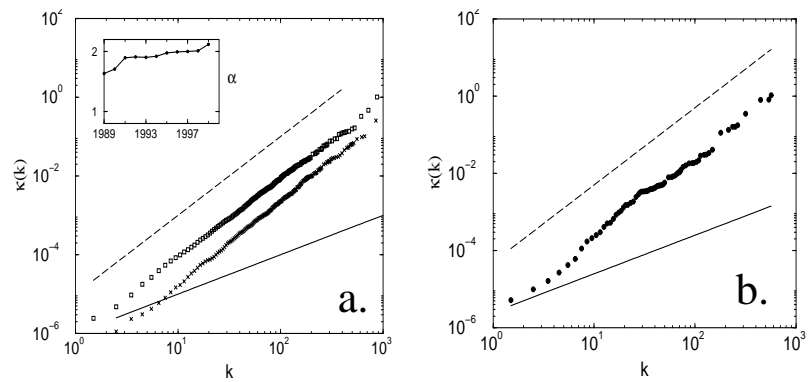

FIG. 1. The $\kappa(k)$ function determined numerically for the citation network (a) and the Internet (b). In (a) the symbols from top to bottom correspond to measurements made at $T_{1}=1991$ and 1995, respectively. For each curve we used $T_{0}=T_{1}-1$. In the inset, we show the measured $\alpha$ exponents for each studied year which was obtained by fitting the whole $\kappa(k)$ curve. For the Internet (b) $\kappa(k)$ was determined using $T_{0}=1999$ and $T_{1}=2000$, yielding $\alpha=1.05$ best exponent. In all measurements $\Delta T=1$ year.
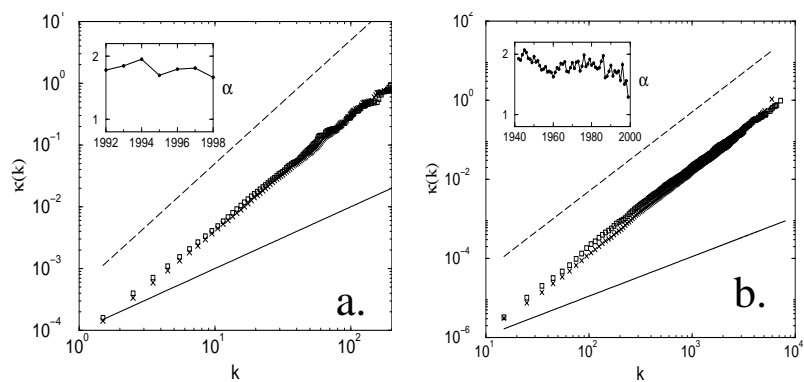

FIG. 2. The $\kappa(k)$ function determined numerically for the NS scientific collaboration (a) and actor network (b). In (a) the symbols from top to bottom correspond to measurements made at $T_{1}=1996$ and 1998, respectively. We have used $T_{0}=T_{1}-1$. In (b) the symbols from top to bottom correspond to measurements made at $T_{1}=1950$ and 1960, respectively. We used as $T_{0}$ nodes, the actors present between 1920 to 1940. In the inset we plotted the measured $\alpha$ exponents for each studied year. In all measurements $\Delta T=1$ year.

On Fig. 1 we present two $\kappa(k)$ curves for the citation network and for the Internet. For both networks we find that the slope of $\kappa(k)$ is very close to two, shown as dashed line on the figure. For the Internet where the measurement was performed for only one year, we obtain $\alpha=1.05$, while for the citation network we determined $\kappa(k)$ for eight different years, obtaining the set of $\alpha$ val- 
ues shown in the Inset, indicating $\langle\alpha\rangle=0.95 \pm 0.1$. Thus we conclude that for these two networks the linear $(\alpha=1)$ preferential attachment hypothesis offers a good approximation.

On the other hand, for the scientific collaboration and actor networks, we find $\alpha<1$ (Fig. 2) The set of $\alpha$ values for these networks are summarized in the insets of Fig. 2. On average we get $\langle\alpha\rangle=0.81 \pm 0.1$ for the actor network, and $\langle\alpha\rangle=0.79 \pm 0.1$ for the scientific collaboration networks.

Internal and external links: The observed sublinear behavior predicts that $P(k)$ for the systems shown in Fig. 2 should follow a stretched exponential [8]. Nevertheless, the measured $P(k)$ indicate that a power law offers a better fit. How can we than reconcile the nonlinear form of $\Pi(k)$ with the measured $P(k)$ ? A potential resolution of this conflict lies in the presence of internal links. For the scientific coauthorship network or the actor web links appear not only from new nodes added to the network, but as a result of new links connecting previously existing nodes as well. The method presented above allows us to investigate separately the attachment mechanisms of these distinct type of links. For is, when determining $\Pi(k)$ we first limit the measurements only to external links, i.e. links that have been added to the system as a result of the appearance of new nodes. Second, we focus only on new internal links, i.e. new links that connect two previously present but disconnected nodes. For example, such internal link appears when two researchers who have not published jointly before, coauthor their first paper together or two actors, who did not act together before, are joined in a new movie. In general, preferential attachment implies that the probability that a new internal link appears between two nodes with $k_{1}$ and $k_{2}$ degree scales with the $k_{1} k_{2}$ product 11. Focusing on the actor network we find that both external and internal links follow preferential attachment. However, the exponent $\alpha$ differs for the two type of links.
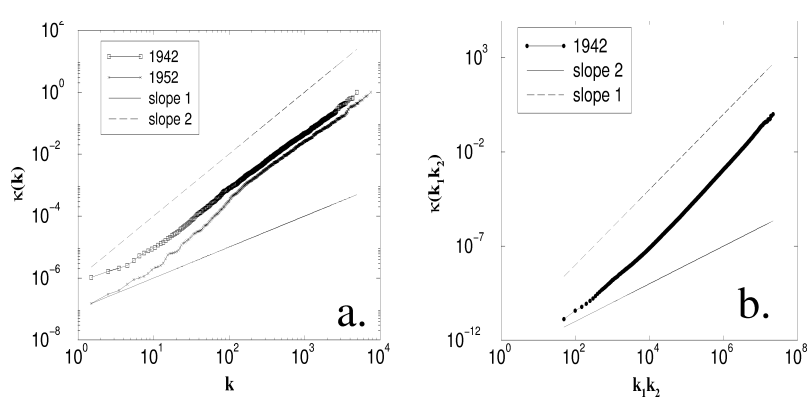

FIG. 3. Preferential attachment of new nodes (a) and new internal links (b) in the actor network. In (b) we plot the $\kappa\left(k_{1} k_{2}\right)$ function. Scaling of $\kappa(k)$ is illustrated for a few selected years. We obtain $0.7<\alpha<1$ for the PA of new nodes (a) and $\alpha$ very close to one for the internal links in the year chosen in figure (b).

From Fig. 3a we conclude that new incoming nodes tend to link to the already existing nodes, following the functional form (2) with $\alpha<1$. The $\kappa\left(k_{1} k_{2}\right)$ function determined from the internal links also follows a power-law. The scaling is clearly not sublinear, and the exponent determined from the assymptotic behavior is close to two (Fig. 3b). Thus the results indicate that the placement of the internal links is also governed by preferential attachment, which scales linearly with $k$. Similar results (not shown) have been obtained for the scientific collaboration web in neuroscience and mathematics [11]. Note that for the science citation network internal links are not allowed, and the data resolution for the Internet does not allows to perform the same analysis at this point. We find that while the preferential attachment of the external links is clearly sublinear, the internal links follow a close to linear behavior. As in both the actor network and the scientific collaboration network the number of internal links far out-weight the external links, we believe that in the asymptotic limit the internal attachment is the one that drives the shape of the $P(k)$ distribution, eventually being responsible for its power-law form. These measurements raise several interesting possibilities for the analytical treatment of the complex coexistence of internal and external links, that could shed further light on the evolution of complex networks.

Initial attractiveness: Dorogovtsev, Mendes and Samukhin have recently suggested that in order to account for the fact that even nodes with no links can acquire links, $\Pi(k)$ should have an additive term, $k_{0}$, called initial attractiveness [6] so that, $\Pi(k) \propto k_{0}+k^{\alpha}$. For $\alpha=1$ it has been demonstrated the the degree exponent, $\gamma$, depends continuously on $k_{0}$. In principle, having the functional form of $\Pi(k)$ allows us to determine $k_{0}$ as well. We inspected the form of $\Pi(k)$, which indeed does indicate that a nonzero $k_{0}$ is present. On the other hand the available statistics was not sufficient to determine unambiguously the value of $k_{0}$. In any case, our estimates indicate that $k_{0}$ is rather small, in the $10^{-6}$ range, thus its presence has no effect on the scaling of $\kappa(k)$ at large $k$. Nevertheless, the nonzero $k_{0}$ plays an important role in starting the evolution of the node connectivity, since in its absence no disconnected node could acquire initial links.

\begin{tabular}{|c||c|c|c|}
\hline Database & $\#$ nodes & \# links & $\alpha$ \\
\hline \hline Citation & 1736 & 83252 & $0.95 \pm 0.1$ \\
\hline Internet & 12409 & 13445 & 1.05 \\
\hline Collaboration & 209293 & 3534724 & $0.79 \pm 0.1$ \\
\hline Actor & 392340 & 33646882 & $0.81 \pm 0.1$ \\
\hline
\end{tabular}

Table 1. Summary of the investigated database, showing the number of nodes, links, and the average value of the obtained exponent $\alpha$.

In summary, our measurement offer direct confirmation for the existence of preferential attachment for rather different real evolving networks. The emerging picture is 
more complex, however, than originally assumed in [4]. We find that for all the networks we studied Eq. (2) gives a good fit for $\Pi(k)$, implying that $\Pi(k)$ follows a power-law. The exponent $\alpha$, however, is system dependent: while for the Internet and the citation network a linear $\Pi(k)$ offers a reasonable fit, for the actor network and collaboration web the attachment rate is sublinear. These results give firmer foundation for the evolving network models, that have been studied extensively to describe the dynamics of real evolving networks. But they also pose an important question: what is the microscopic origin of preferential attachment? What determines the exponent $\alpha$ in general? While some preliminary answers have been proposed 13,14, a good understanding of this fundamental question is still lacking.

We acknowledge useful discussion with R. Albert, I. Derényi, and T. Vicsek. This research was supported by NSF, PHY-9988674 and CAREER DMR97-01998.

* on leave from: Babes-Bolyai University, Dept. of Theoretical Physics, str. Kogalniceanu 1, RO-3400, ClujNapoca, Romania.

[1] D.J. Watts, Small World (Princeton University Press, Princeton, 1999); S. H. Strogatz, Nature 410, 268 (2001); M.E.J. Newman, J. Stat. Phys. 101, 819 (2000)

[2] D. J. Watts and S.H. Strogatz, Nature 393, 440 (1998)

[3] R. Albert, H. Jeong and A.-L. Barabási, Nature 401, 130 (1999)

[4] A.-L. Barabási and R. Albert, Science 286, 509 (1999); A.-L. Barabási, R. Albert and H. Jeong, Physica A 272, 173 (1999)

[5] R. Cohen, K. Erez, D. ben-Avraham and S. Havlin, Phys. Rev. Lett. 85, 4626 (2000)

[6] S. N. Dorogovtsev, J.F.F. Mendes and A. Samukhin, Phys. Rev. Lett. 85, 4633 (2000)

[7] F. Slanina and M. Kotrla, Phys. Rev. E 62, 6170 (2000)

[8] P.L. Krapvisky, S. Redner and F. Leyvraz, Phys. Rev. Lett. 85, 4629 (2000)

[9] L. Kullmann and J. Kertész, cond-mat/0012410

[10] A.-L. Barabási, R. Albert and H. Jeong, Physica A 281, $69(2000)$

[11] A.-L. Barabási, H. Jeong, Z. Néda, E. Ravasz, A. Schubert and T. Vicsek, preprint 2001

[12] M.E.J. Newman Proc. Natl. Acad. Sci. USA, 98, 404 (2001); cond-mat/0007214

[13] A. Vazquez, cond-mat/0006132

14] N. Dorogovtsev, J.F.F. Mendes and A. Samukhin, condmat/0011115

[15] P. Erdős and A. Rényi, Publicationes Mathematicae 6, 290 (1959); P. Erdős and A. Rényi, Acta Math. Sci. Hung. 12, 261 (1961)

[16] B. Bollobás, Random Graphs (Academic, London, 1985)

[17] http://www.webofscience.com

[18] http://www.imdb.com 